\title{
Phonon Scattering at Surfaces and Interfaces
}

\author{
W. Eisenmenger \\ Physikalisches Institut, Universität Stuttgart, Pfaffenwaldring 57, \\ D-7000 Stuttgart 80, Fed. Rep. of Germany
}

\section{Introduction}

Since the discovery of the thermal boundary resistance by Kapitza /1/ in 1941, there have been extensive experimental and theoretical studies on the underlying phonon transmission, reflection and scattering processes at surfaces and interfaces. The field has been recently reviewed by A.C. Anderson /2/ and A.F.G. Wyatt $/ 3 /$. The reader is referred to these authors, since the present contribution cannot give a complete account of all the relevant work done. For ideal solid-liquid or solid-solid boundary conditions the theoretical treatment according to the acoustic mismatch model by Khalatnikov /4/ and Little /5/ predicts strong phonon reflection at the solid-liquid $4 \mathrm{He}$ interface, but weak phonon reflection for most solid-metal interfaces. In the latter case the acoustic model was especially successful for the calculation of phonon spectra and intensity distributions emitted by metallic heaters into dielectric substrates as performed by Weis and co-workers /6/ taking also account of phonon focussing $17 \%$. Experimental evidence for the applicability of the acoustic model to the solid-liquid ${ }^{4} \mathrm{He}$ interface for phonon frequencies ranging from 100 to about $700 \mathrm{GHz}$ was provided by the famous UHV cleaving experiments of Kinder and co-workers /8/. More recently Basso et al. /9/ demonstrated that phonons of $100 \mathrm{GHz}$ are almost ideally reflected from $\mathrm{Si}-1$ iquid ${ }^{4} \mathrm{He}$ interfaces after laser annealing the Si-crystal under UHV conditions. Even multiple solid-solid interfaces as in the phonon transmission experiment through a GaAs:AlGaAs MBE superlattice by Narayanamurti et al. /10/ exhibit ideal acoustic properties in the frequency regime of $200 \mathrm{GHz}$. Similar results have been obtained with amorphous $\mathrm{Si} / \mathrm{SiO}_{2}$ superlattices by Koblinger et al. $/ 11 /$.

In contrast to these examples of "ideal" acoustic behaviour, many "real" solid-liquid or solid-solid interfaces deviate significantly from the acoustic model, as well known by the anomalous high phonon transmission /Kapitza anomaly/ from solids into liquid He cf. /3/ or by phonon losses /12/ and anomalous high phonon backscattering $/ 13 /$ at solid-solid interfaces. Phonon transmission and phonon reflection experiments with nonideal acoustic interface systems demonstrated in addition to specular diffraction or reflection large contributions of diffusely transmitted and or scattered phonons cf. $/ 3 /, / 14 /, / 15 /$. This is consistent with the fact that violation of the k-parallel conservation leads to additional channels of phonon transport cf. $/ 3 /$. The diffuse backscattering from uncovered crystal surfaces is strongly reduced by contact with liquid $\mathrm{He} / 16 /, / 17 /$, whereas the specular reflected components remain almost unchanged. Therefore, it appears natural to assume that the same nonideal interface properties leading to diffuse backscattering from the uncovered surface are mainly responsible for anomalous high phonon transmission into the $4 \mathrm{He}$ bath or for enhanced backscattering at solid-solid interfaces.

The following report on experiments with frequency-dependent phonon backscattering at differently prepared $\mathrm{Si}$ and $\mathrm{Al}_{2} \mathrm{O}_{3}$ surfaces demonstrates the variety of phenomena in phonon backscattering of real surfaces to be explained 
by appropriate models. This work has been performed by $\mathrm{S}$. Burger in co-operation with K. Laßmann, S. Döttinger, E. Mok, R. Schneider and the author.

\section{Experiment}

In the experimental arrangement of Fig. I a Si-crystal is sealed against an UHV chamber, carrying also a window for laser annealing experiments $/ 18 /$ to be described later. The main phonon propagation path for $90^{\circ}$ specular reflection corresponds to maximum phonon focussing of the $\mathrm{ft}$-mode, the same holds for the paths of diffuse scattering and mode conversion. The geometry is well suited for differentiating between various phonon paths by reflection and scattering in pulse experiments. For comparison it is possible to perform theoretical Monte Carlo simulations of the expected pulse signal $/ 13 /$. For phonon generation mostly constantan heaters have been used with frequency variation by changing the heater power. The main frequency range could be selected with Sn-tunneling junctions at $280 \mathrm{GHz}$ and $\mathrm{Al}$-junctions with a lower frequency limit of roughly $80 \mathrm{GHz} / 19 /$ as detectors.

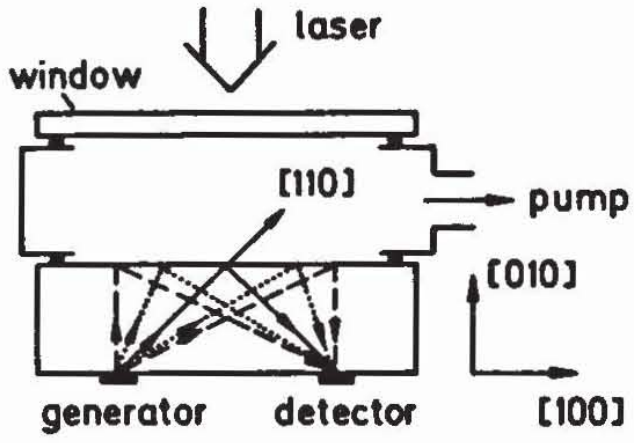

Fig. I UHV sample chamber with crystal and optical window for laser annealing. The propagation paths are: Full lines: specular and diffuse longitudinal mode, first peak in Fig.2. Specular and diffuse fast transverse mode, third peak in Fig. 2 . Dotted lines: mode conversion longitudinal fast transverse phonons, second peak in Fig.2. Dashed lines: diffuse scattered fast transverse phonons along phonon focussing direction, fourth peak in Fig.2.

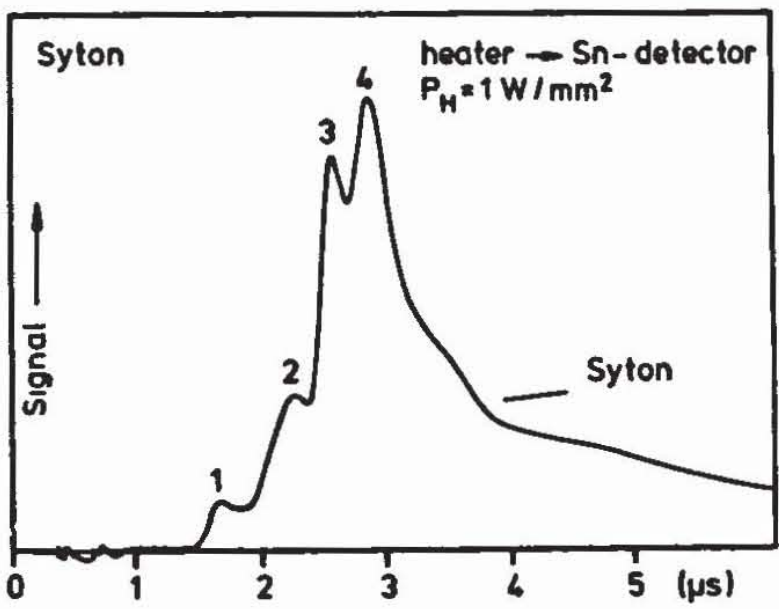

Fig. 2 Phonon backscattering signal from a Syton-polished, uncovered Si-surface at $280 \mathrm{GHz}$. The scattering is almost completely diffuse, see text. For peak identification cf. Fig.l.

\section{Phonon backscattering from mechanical and chemical polished Silicon}

The crystals were prepared by polishing with 9 um diamond and finally with Syton. Fig. 2 shows the Sn-detector pulse response /19/ corresponding to $285 \mathrm{GHz}$ with high heater generator power. This agrees with earlier results of Marx /20/ indicating almost complete diffuse phonon backscattering as evidenced by comparison with the computer simulated pulse response. A more direct check was the preparation of real "rough" surfaces with $0,25 \mu \mathrm{m}$ or even $15 \mu \mathrm{m}$ diamond showing agreement with the result of the Syton polished surface. 


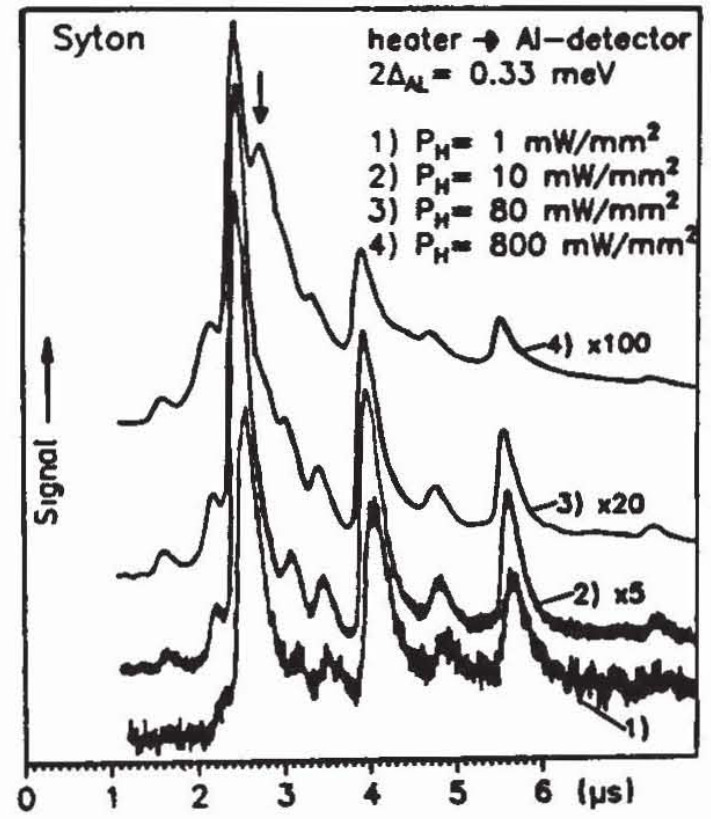

Fig. 3 Backscattering signal changing from specular to diffuse of a Syton-polished Si-surface for different heater power with an $A 1$-junction as detector. The arrow at curve 4 marks the diffuse scattering contribution at high heater power.

A dramatic change of the backscattering signal (Fig. 3) is observed with an Al-detector sensitive also for low frequencies in the $80 \mathrm{GHz}$ range. The observed echoes result from oblique multiple phonon reflection between the crystal face carrying generator and detector and the opposing surface. This gives direct evidence for specular phonon reflection especially at low heater power. With increasing heater power and the resulting spectral shift to higher frequencies again the most prominent diffuse scattering peak no. 4 (cf. Fig.1) marked by the arrow is clearly observed. This is supported by the influence of 4 He coveraqe shown in Fig. 4 and 5 . With low heater power the signal reduction in Fig. 4 is almost unobservable, whereas at high heater power the significant signal reduction in Fig. 5 corresponds to a strong diffuse backscattering contribution. Polishing only with diamond $0,25 \mu \mathrm{m}$ grain size reduces the specular reflection as expected, but at low heater power the influence of the 4he coverage is surprisingly smail. Apparently the polishing grain size of $0,25 \mu \mathrm{m}$ is too small for strong diffuse scattering at this frequency. The

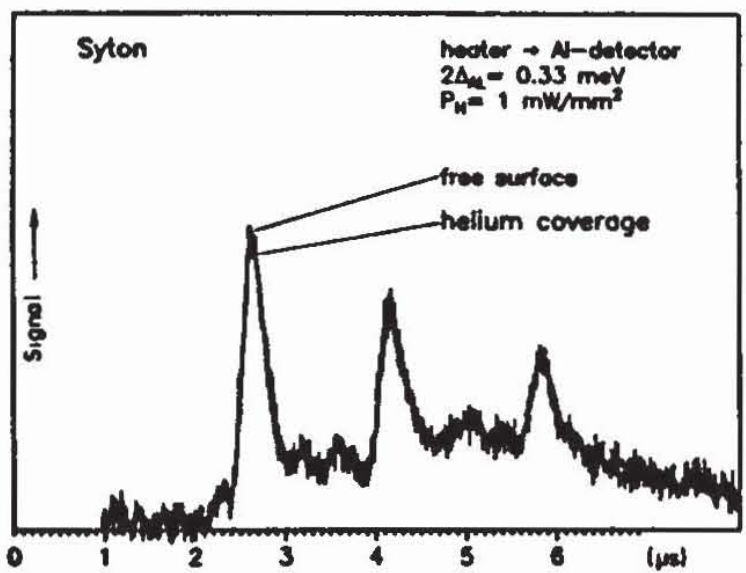

Fig. 4 Backscattering signal for a Syton-polished Si-surface at low heater power without and with liquid $4 \mathrm{He}$ contact.

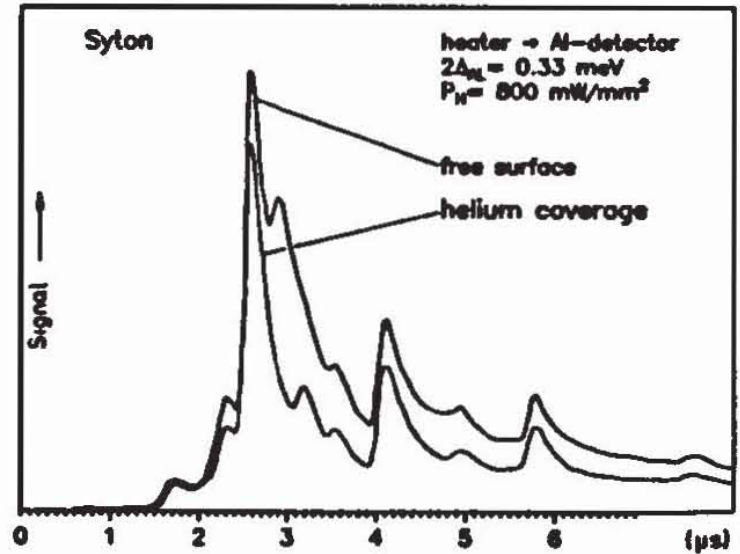

Fig. 5 Backscattering signal for a Syton-polished Si-surface at high heater power without and with liquid ${ }^{4} \mathrm{He}-$ contact. 


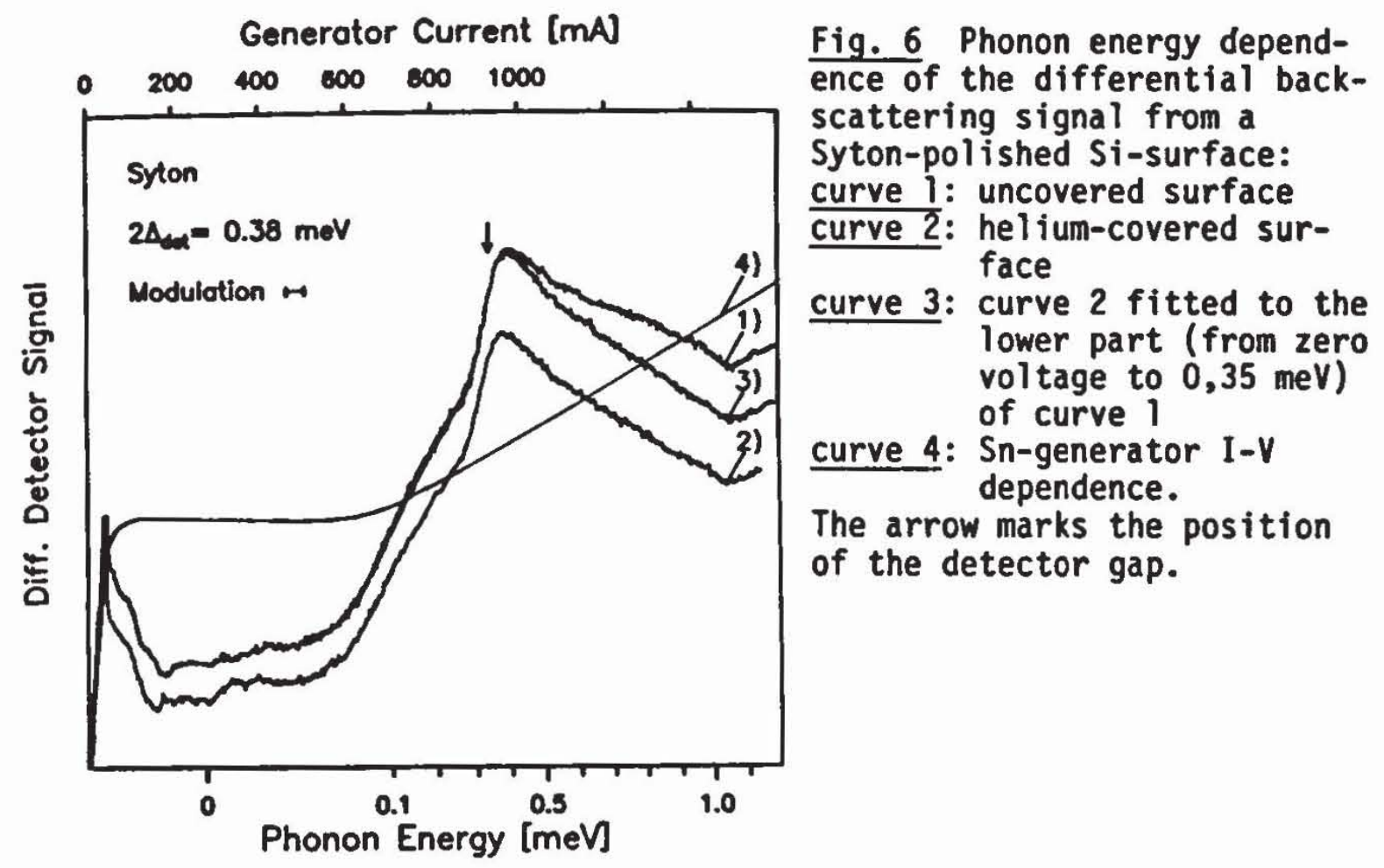

crystal surface showed residual fine scratches under high optical resolution microscopy, whereas the Syton polished surface appeared optically smooth. Increasing the heater power to $1 \mathrm{~W} / \mathrm{mm}^{2}$ the diamond polished surface showed with Al-detection strong diffuse scattering almost identical to Fig. 2 with corresponding signal reduction by liquid 4 He contact.

More detailed information on the frequency dependence of the phonon scattering has been obtained with a Sn-generator (and an Al-detector) as a tunable phonon source using the modulation technique. From intensity reasons pulse resolution was not obtained and the result for a Syton polished Si-surface in Fig. 6 represents a signal-time average. Curve 1 and curve 2 correspond to the frequency dependence of phonon backscattering without and with liquid $4 \mathrm{He}$ coverage respectively. Curve 3 is the same signal as in curve 2, but normalized to the maximal value of curve 1. Both curves being almost identical below phonon energies of $0,3 \mathrm{meV}$ show a signal reduction of curve 3 relative to curve 1 above $0,35 \mathrm{meV}$. This indicates an increasing phonon escape from the solid into the $4 \mathrm{He}$ liquid with a threshold of $0,35 \mathrm{meV}$ in agreement with the frequency dependence $/ 21 /$ of the phonon escape from a Sn-tunnel junction into liquid $4 \mathrm{He}$. This substrate independence is an indication for the more general nature of the $0,35 \mathrm{meV}$ escape threshold which consequently may be related to surface impurities or defects in combination with liquid 4 He boundary states $122 /, 123 /$.

\section{Phonon backscattering from Silicon treated by laser annealing}

Since mechanical, chemical and other polishing procedures did not change the almost complete diffuse backscattering of $285 \mathrm{GHz}$ phonons into at least partly specular reflection, the laser annealing method $/ 9 /$ has been applied also to our sample configuration /18/ (see Fig. 1) with a Sn-detector. In using a Q-switch ruby laser with $1 \mathrm{~J}$ pulse power and $50 \mathrm{~ns}$ duration, it was sufficient to treat the effective scattering surface area of the Si-crytal with 5 laser pulses until reaching the maximal and final change to specular reflection shown in Fig. 7. Again covering the scattering surface with liquid 4 He results 


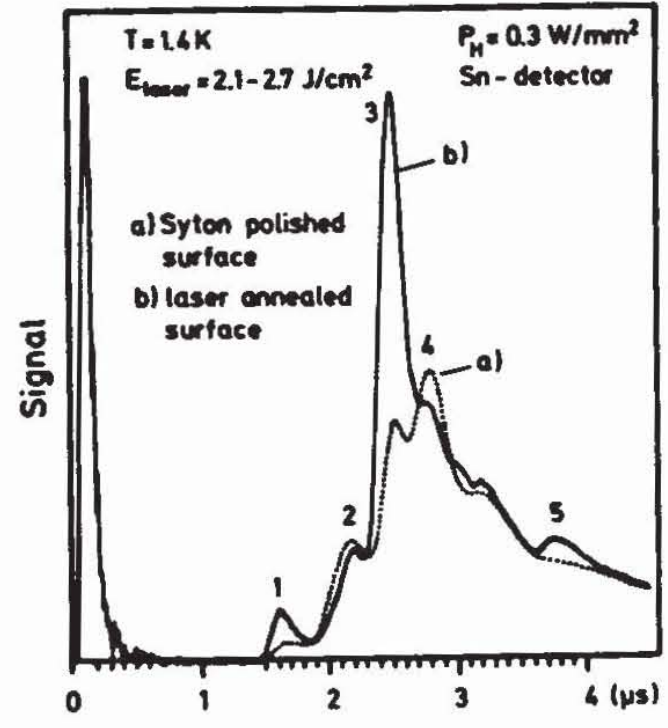

Fig. 7 Phonon backscattering signal of a polished Si-surface before (curve a) and after (curve b) laser annealing. Besides the increase of pulse 3 by specular reflection also a double specular reflection signal 5 is observed.

in only a $13 \% \mathrm{ft}$ signal reduction. This demonstrates the high degree of specular phonon refiection of the Si-surface treated by laser annealing even at the frequency of $285 \mathrm{GHz}$. Exposing the reflecting surface to air (at room temperature) changed the reflection again back into complete diffuse scattering with corresponding signal reduction by liquid 4 He contact. Reflection could only be obtained anew with pulse powers of the original value, i.e. surface melting. The laser annealing method /9/ also in our experiments at $285 \mathrm{GHz}$ proved as a very efficient means of ideal acoustic surface preparation.

The residual diffuse scattering observed at $285 \mathrm{GHz}$ decreases as to be expected with frequency $/ 24 /$. The experimental accuracy, however, has to be improved to resolve the $0,35 \mathrm{meV} / 21 /$ phonon escape threshold.

\section{Phonon backscattering from Sapphire surfaces}

Specular and diffuse reflection at sapphire surfaces has been already observed by Taborek and Goodstein $/ 17 /$. Experiments with the sample arrangement of Fig. 1 and the sapphire surface in the (001) plane /25/ indicated by comparision with Monte Carlo computations a ratio of $3: 4$ between specular and diffuse scattering in the $80 \mathrm{GHz}$ frequency range. The scattering surface was diamond polished with a final Syton treatment. Surprisingly a change to almost complete specular reflection (Fig. 8 ) could be obtained by a 10 min. polishing

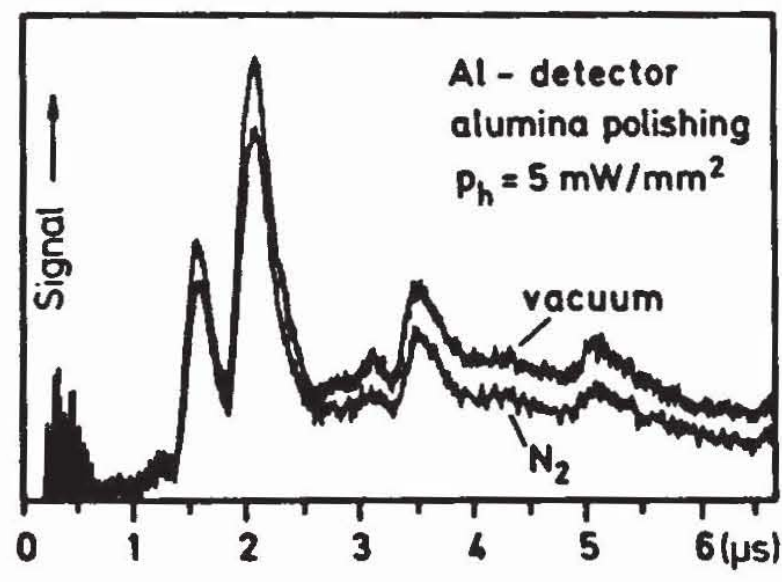

Fig 8 Specular backscattering of a Sapphire surface polished with cubic $\mathrm{Al}_{2} \mathrm{O}_{3}$ without and with condensed $\mathrm{N}_{2}$. 
with cubic $\mathrm{Al}_{2} \mathrm{O}_{3}$ of $50 \mathrm{~nm}$ grain size. This is demonstrated by multiple reflection signals and by only a small signal reduction with condensed $\mathrm{N}_{2}$ or liquid 4 He contact. If the surface is treated again a few minutes with Syton, the original contribution of diffuse phonons is observed, correspondingly the specular reflection is found by a succeeding $\mathrm{Al}_{2} \mathrm{O}_{3}$ polish. This has been repeated several times. The corresponding changes of the surface properties are possibly related to structural or chemical differences on the nanometer scale. But electron micrographs of surface replicas did not show different surface structures within $10 \mathrm{~nm}$ resolution.

\section{Phonon scattering from condensates on ideal surfaces}

With primarily ideal reflecting surfaces it is now possible to study the phonon scattering by controlled adsorption of atoms or molecules /26/ or condensed layers. The result of an experiment /27/ with a $324 \mathrm{~nm}$ constantan film evaporated on a Syton polished Si-crystal is shown in Fig. 9. At a frequency of $80 \mathrm{GHz}$ the specular reflection of the uncovered surface is almost completely changed to strongly reduced diffuse backscattering. This is in accord with the acoustic model. Almost perfect acoustic matching results in high transmission to the metal film. Phonon absorption and emission by electron interactions results in themalization and subsequent diffuse emission back into the substrate. Similar experiments by Klitzner and Pohl /28/ on the temperature dependence of heat transport in perfect single crystal rods covered by different adsorbates and thin filmsexhibit a significant frequency (temperature) dependence related in a characteristic way to the film structure.

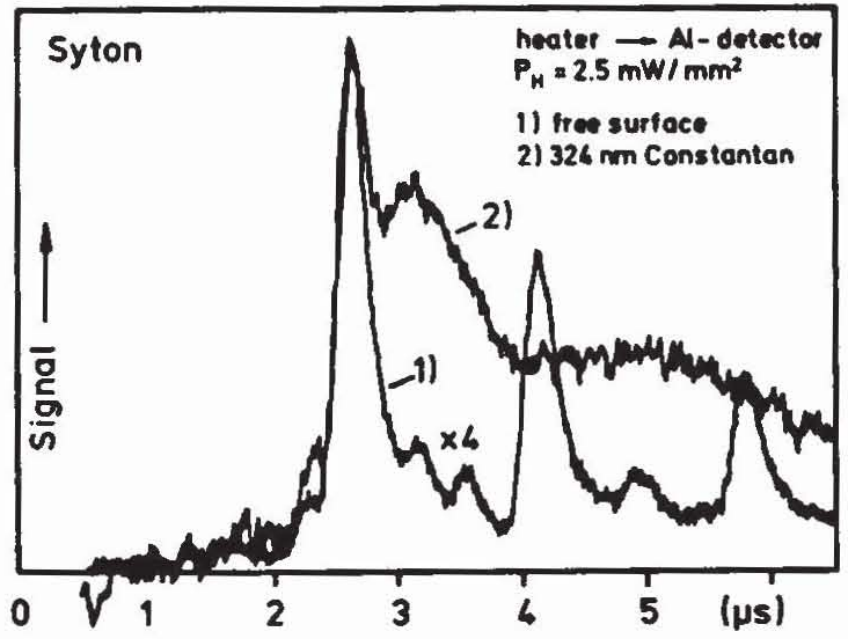

Fig. 9 Phonon backscattering from a Syton-polished Si-surface before (curve 1) and after (curve 2) evaporation of $324 \mathrm{~nm}$ thick constantan film. Curve 1 is reduced in amplitude by a factor of four.

\section{Conclusion}

Several experimental techniques as UHV cleaving $/ 8 /$, laser annealing $/ 9 /$, MBE /10/ or other UHV evaporation techniques /11/ or even the simple polishing procedures ( $100 \mathrm{GHz}$ range) reported here are presently available for the preparation of acoustically ideal surfaces and interfaces. For these systems the classical acoustic models appear to be applicable up to several $100 \mathrm{GHz}$. Deviations from the acoustic model as the Kapitza anomaly are accompanied by diffuse phonon backscattering and or transmission. Only little is known under what conditions these scattering processes are elastic or inelastic. For uncovered Si-surfaces $/ 12 /$ elastic diffuse scattering has been observed. Diffuse phonon transmission into liquid 4 He appears to be accompanied by 
inelastic decay, cf. ref. $/ 3 /$, as has been found also in phonon loss processes $112 /$ at the interface between different solids as e.g. tunneling junctions and substrates or other systems.

A large variety of models cf. also ref. /3/ has been proposed in order to explain anomalous or nonacoustical properties. These models are based in principle on diffuse scattering (violation of the $k=$ conservation) by surface roughness $/ 31 /$, surface, subsurface or interface defects, adsorbed atoms, molecules and corresponding phonon states in the form of two-level systems, either at the surface $/ 22 /$ or in the first ajoining 4 He layer $/ 23 /$. Similar phonon states or nonlinear resonant systems are possible in adsorbed atomic or molecular clusters or islands of adsorbates $/ 21 /, / 19 /, / 26 /, / 28 /, / 29 /, / 32 /$, for which already experimental evidence has been given by a characteristic frequency dependence of the diffuse scattering processes. It is to be hoped that different mechanisms for diffuse acoustic phonon scattering at surfaces and interfaces can in future be identified by their frequency dependence and by other significant properties, as for example the polarisation and angle dependence $130 \%$.

I should like to thank all my collaborators for their contributions and numerous stimulating discussions. I am also grateful to Tom Klitzner and R.0. Pohl as well as to A. Khater for supplying preprints of their recent results. This work was supported by the Deutsche Forschungsgemeinschaft.

\section{$\underline{\text { References }}$}

/1/ P.L. Kapitza, J. Phys., USSR, 4, 181 (1941)

12/ A.C. Anderson in Nonequilibrium, Superconductivity, Phonons and Kapitza Boundaries; K.E. Gray Ed., Nato Adv.Study, Inst. Series B, Physics, Vol.65, Plenum Press, New York, 1981

/3/ A.F.G. Wyatt, same source as /2/

14/ I.M. Khalatnikov, Zh. eksp. theor. Fiz. (USSR) 22:687 (1951)

15/ W.A. Little, Can. J. Phys. 37, 334 (1959)

/6/ F. Rösch, 0. Weis, Z. f. Phys., B 27, 33 (1977)

/7/ B. Taylor, H.J. Maris, C. Elbaum, Phys. Rev. Lett. 23, 416 (1969)

18/ J. Weber, W. Sandmann, W. Dietsche, H. Kinder, Phys.Rev. Lett 40, 1469 (1978)

19/ H.C. Basso, W. Dietsche and H. Kinder, P. Leiderer in Phonon Scattering in Condensed Matter (W. Eisenmenger, K. LaBmann, S. Döttinger Ed.)

Springer Series in Solid State Sciences, 51

/10/ V. Narayanamurti, H.L. Störmer, M.A. Chin, A.C. Gossard. W. Wiegmann, Phys. Rev. Lett. 43, 2012 (1979)

$111 / 0$. Koblinger, J. Mebert, E. Dittrich, S. Döttinger, W. Eisenmenger, this volume and Verhandlungen d. DPG 5, 1481 (1986)

$112 /$ L.J. Challis, in Phonon Scattering in Condensed Matter,

W. Eisenmenger, K. Laßmann, S. Döttinger Ed., Springer Series in Solid State Sciences, Vol 51, 2, (1984)

H.J. Trumpp, W. Eisenmenger, Z. Phys. B 28, 159 (1977)

/13/ D. Marx, W. Eisenmenger, Phys. Lett. 82 A, 291 (1981)

/14/ C.J. Guo, H.J. Maris, Phys. Rev. Lett $\frac{29}{6}, 855$ (1972)

$115 /$ R.E. Horstmann, J. Wolter, Phys. Lett. $62 \mathrm{~A}, 279$ (1977)

$116 /$ J.T. Fol insbee, J.P. Harrison, J. of Low Temp. Phys. 32, 469 (1978)

D. Marx, J. Buck, K. LaBmann, W. Eisenmenger, J. Phys. C6, suppl. to No. 8, 1015 (1978)

/17/ P. Taborek, D.L. Goodstein, J. Phys. C12, 4737 (1979);

Sol. State Comm. 38, 215 (1981); Phys. Rev. B22, 1550 (1980)

118/ E. Mok, S. Burger, S. Döttinger, K. LaBmann, W. Eisenmenger, Phys. Lett. 114, 473 (1986) 
/19/ S. Burger, K. LaBmann, W. Eisenmenger, J. of Low Temp. Phys.61, 401 (1985)

/20/ D. Marx, W. Eisenmenger, Phys. Lett. 93A, 152 (1983);

2. Phys. B48, 277 (1982)

121/ 0. Koblinger, U. Heim, M. Welte, W. Eisenmenger, Phys.Rev.Lett.51, 284 (1983); a slightly different frequency dependence was earlier found by E.S. Sabisky, C.H. Anderson, Sol. State Comm. 17, 1095 (1975)

/22/ H. Kinder, Physica 107B, 549 (1981)

123/ T. Nakayama, Phys. Rev. B32, 777, (1985); J. Phys. C18, 667 (1985)

/24/ R. Schneider, Diplomarbeit, Universität Stuttgart, $\overline{986}$

/25/ S. Burger, W. Eisenmenger, K. LaBmann, Proc.LT17, Vol. 9A, North Holland (1984)

/26/ L. Köster, St. Wurdack, W. Dietsche, H. Kinder, this volume and Verhandlungen d. DPG 5, 1477 (1986)

127/ S. Burger, Dissertation, Universität Stuttgart (1986)

/28/ R.0. Poh1 and B. Stritzker, Phys. Rev. B25, 3608 (1982);

T. Klitzner and R.O. Pohl in Phonon Scattering in Condensed Matter ed.

by W. Eisenmenger, K. Laßmann and S. Döttinger (Springer, Berlin, 1984)

p. 188; T. Klitzner and R.0. Pohl this volume

/29/ A. Khater, Phys. Rev. B (submitted)

/30/ H. Kinder, A. de Ninno, D. Goodstein, G. Paterno, F. Scaramuzzi and

S. Cunsolo,, Phys. Rev. Lett. 55, 2441 (1985) and this volume

/31/ N.S. Shiren J. Phys. Supp1. C6, 42, 816, (1981),

Phys. Rev. Lett. 47, 1466, (1982)

/32/ L.J. Challis, J. Phys. C7, 481, (1974) 